Discussion/conclusion By concentrating activity in venues with higher positivity, in line with guidance from the NCSP, it has been possible to achieve the DRI target whilst working within tighter economic constraints. In particular, outreach screening was costly and produced low volumes of tests with low positivity.

\section{O22 PERFORMANCE OF THE BD MAXTM CT/GC/TV ASSAY FOR DETECTION OF CHLAMYDIA, GONORRHOEA AND TRICHOMONAS}

${ }^{1}$ Barbara Van Der Pol*, ${ }^{1}$ Grace Daniel, ${ }^{2}$ James Williams, ${ }^{2}$ DeAnna Fuller, ${ }^{2}$ Thomas Davis, ${ }^{3}$ Stephanie Taylor, 'Edward Hook. 'University of Alabama at Birmingham, Birmingham, AL, USA; ${ }^{2}$ Indiana University School of Medicine, Indianapolis, IN, USA; ${ }^{3}$ Louisiana State University Health Sciences Center, New Orleans, LA, USA

\subsection{6/sextrans-2015-052126.22}

Background Chlamydia, gonorrhoea and trichomonas infections remain highly prevalent with annual WHO estimates of 106, 107 and 276 million cases respectively. Screening for all 3 infections in a single assay could improve control efforts.

Aim This study assessed the performance of the BD MAX ${ }^{\mathrm{TM}} \mathrm{CT} /$ GC/TV (BD MAX) for detection of chlamydia, gonorrhoea and trichomonas DNA compared to routine diagnostic methods.

Methods Urine, patient-collected vaginal and endocervical specimens were obtained from 1854 women. BD MAX assay results were compared to TV culture (InPouch), TV wet mount, Aptima $\mathrm{AC} 2$ and TV assays and the BD Viper ${ }^{\mathrm{TM}} \mathrm{CTQ}^{\mathrm{x}} / \mathrm{GCQ}^{\mathrm{x}}$ assays.

Results Prevalence for chlamydia, gonorrhoea and trichomonas was $7.3,2.3$ and $14.7 \%$, respectively. Sensitivity estimates ranged from 92.2-99.2, 94.9-95.1 and 92.9-96.1 for chlamydia, gonorrhoea and trichomonas, respectively. Specificity estimates for each test were $\geq 98.6$. Of the 128 out of 1758 (7.3\%) women with chlamydia infections, concomitant gonococcal and trichomonal infections were present in 11.7 and $12.5 \%$, respectively. The sensitivity of the assay for chlamydia when co-infections were present ranged from 92.6-96.1\%. Similarly the sensitivity of the gonorrhoea and trichomonas detection was not affected by the presence of concomitant chlamydial infections with estimates ranging from $93.8-100 \%$ and $89.5-100 \%$, respectively.

Discussion The performance of the BD MAX assay was similar to that of other molecular diagnostic assays. A substantial proportion of women with chlamydia are co-infected with gonorrhoea and/or trichomonas. Trichomonas was more prevalent than chlamydia and gonorrhoea combined. Detection of all three infections in a single assay may improve identification and treatment of these STI.

\section{O23 PORA PSEUDOGENE DELETION AMONGST NEISSERIA GONORRHOEAE ISOLATES FROM THE GONOCOCCAL RESISTANCE TO ANTIMICROBIALS SURVEILLANCE PROGRAMME (GRASP)}

${ }^{1}$ Martina Toby, ${ }^{2}$ Pamela Saunders, ${ }^{2}$ Michelle Cole, ${ }^{2}$ Vlad Grigorjev, ${ }^{2}$ Sarah Alexander*, ${ }^{2}$ Cathy Ison. 'Guys and St Thomas' NHS Foundation Trust, London, UK; ${ }^{2}$ Public Health England, London, UK

\subsection{6/sextrans-2015-052126.23}

Background/introduction In the last four years, isolates of N. gonorrhoeae have been identified in Australia, Sweden,
Scotland and England which lack the gonococcal porA pseudogene and consequently result in negative results in the diagnostic porA pseudogene real-time-PCR (RT-PCR) for N. gonorrhoeae.

Aim(s)/objectives This study sought to determine the prevalence of porA pseudogene negative isolates amongst isolates received at Public Health England (PHE) through the national gonococcal resistance to antimicrobials surveillance programme (GRASP).

Methods DNA lysates were prepared from 533 N. gonorrhoeae isolates received from 20 centres via GRASP during 2011. Any isolate with a RT-PCR por A pseudogene negative result was repeated from a fresh culture and the porA gene was additionally DNA sequenced. Isolates were additionally tested using the gonococcal opa gene RT-PCR.

Results Four isolates $(4 / 533,0.8 \%)$ were found to be reproducibly negative with the porA pseudogene RT-PCR, but were positive with opa gene RT-PCR. DNA sequencing determined that two isolates contained the Neisseria meningitidis porA gene. Both isolates were from patients attending a clinic in SouthLondon.

Discussion/conclusion Less than one percent of the GRASP isolates from patients attending clinics across England expressed the meningococcal porA gene and therefore tested negative on the in-house porA assay. The low prevalence indicates that these isolates do not present a major diagnostic or public health problem. However, microbiologists should remain vigilant for any isolates giving anomalous results and when using the porA pseudogene RT-PCR consider multiplexing it with the opa-gene RT-PCR.

\section{O24 CONFIRMING GC NAAT RESULTS: IS IT ALWAYS NECESSARY?}

Sarah Alexander, Hemanti Patel, Simon Warwick, Helen Fifer*. Public Health England, London, UK

\subsection{6/sextrans-2015-052126.24}

Introduction Current guidance recommends that all specimens testing positive using a N. gonorrhoeae Nucleic Acid Amplification Test (GC NAAT) be confirmed using a second test with an alternative target, in order to achieve a positive predictive value above $90 \%$.

Aim To determine rates of GC NAAT confirmations by primary screening test and specimen site.

Methods 994 specimens which were GC NAAT positive at local laboratories were sent for confirmation using an in-house multiplex PCR with PorA and opa gene targets. A correlation between the confirmatory real-time PCR results, specimen site and GC screening NAAT was undertaken. For the purposes of this analysis equivocal results were regarded as positive and inhibited results were excluded.

Results Overall, $57 \%$ of specimens examined could be confirmed as GC positive using the in-house real-time PCR test (Table 1).

Discussion High rates of confirmation can be achieved when examining genital, rectal and urine specimens irrespective of the GC screening NAAT. However $>90 \%$ confirmatory rates were only achieved when examining male urine specimens which had been screened using the Probetec and Cobas Amplicor tests, 
although caution should be applied if extrapolating this data to low prevalence settings. Poor confirmation rates from throat specimens is probably due to cross-reactivity with commensal Neisseria, and highlights confirmation is essential when testing these samples.

\begin{tabular}{|c|c|c|c|c|}
\hline & $\begin{array}{l}\text { Genital Swab } \\
\text { (Female) } \\
{[\mathrm{n}=119]}\end{array}$ & $\begin{array}{l}\text { Urine } \\
\text { (Male) } \\
{[n=84]}\end{array}$ & $\begin{array}{l}\text { Rectal } \\
{[n=97]}\end{array}$ & $\begin{array}{l}\text { Throat } \\
{[n=694]}\end{array}$ \\
\hline $\begin{array}{l}\text { Probetec GC } \\
\text { Qx (Becton } \\
\text { Dickinson) }\end{array}$ & $78.7 \%(37 / 47)$ & $94 \%(47 / 50)$ & $85.1 \%(23 / 27)$ & $44.2 \%(248 / 587)$ \\
\hline $\begin{array}{l}\text { Cobas } \\
\text { Amplicor } \\
\text { (Roche) }\end{array}$ & $83.3 \%(50 / 60)$ & $91.3 \%(21 / 23)$ & $79.4 \%(27 / 34)$ & $48.2 \%(27 / 56)$ \\
\hline $\begin{array}{l}\text { RealTime } \\
\text { CT/NG (Abbott) }\end{array}$ & $83.3 \%(10 / 12)$ & $72 \%(8 / 11)^{*}$ & $80.5 \%(29 / 36)$ & $88.2 \%(45 / 51)$ \\
\hline
\end{tabular}

\section{O25 USE OF CEFTRIAXONE AND DOXYCYCLINE WHEN TREATING GONORRHOEA: IS IT PRESCRIBED APPROPRIATELY?}

John Were*, Katy Town, Hamish Mohammed, John Saunders, Stephanie Chisholm, Helen Fifer, Gwenda Hughes. Public Health England, London, UK

\subsection{6/sextrans-2015-052126.25}

Background National gonorrhoea treatment guidelines recommend ceftriaxone with azithromycin as first-line therapy, but doxycycline is recommended instead of azithromycin for patients with gonococcal pelvic inflammatory disease (PID). In 2013, $86.5 \%$ of patients in the Gonococcal Resistance to Antimicrobials Surveillance Programme (GRASP) were treated with the recommended therapy, but $3.9 \%$ were treated with doxycycline instead of azithromycin.

Objectives The objective of this analysis was to determine whether ceftriaxone plus doxycycline were prescribed for appropriate indications.

Methods Using GRASP 2013 data, patients prescribed the recommended therapy were compared with patients prescribed ceftriaxone and doxycycline, and associations were assessed using univariate and multivariate logistic regression.

Results In 2013, of the 913 patients prescribed ceftriaxone and azithromycin, $45.9 \%$ were men who have sex with men (MSM), $20 \%$ were women and $34.1 \%$ were heterosexual men while, of the 45 patients prescribed ceftriaxone and doxycycline, $64.4 \%$ were MSM, 28.9\% were women and 6.7\% were heterosexual men $(\mathrm{p}=0.001)$. Of those prescribed ceftriaxone and doxycycline, 22.2\% were MSM with chlamydia co-infection and 17.7\% were women with PID. On multivariate analysis, MSM coinfected with chlamydia (aOR 3.4, 95\% CI, 2.5-4.6; $\mathrm{p}=0.001$ ) and women diagnosed with gonococcal PID (OR,144.8, 95\% CI, 24.2-864.0; $\mathrm{p}<0.001)$ were more likely to be prescribed ceftriaxone and doxycycline.

Conclusion Less than a fifth of prescriptions for ceftriaxone with doxycycline were issued to treat gonococcal PID. Use of ceftriaxone with doxycycline may be preferred to treat MSM coinfected with chlamydia by some clinicians. However, as levels of tetracycline resistance in gonorrhoea are high, this may not provide the dual treatment coverage required.
026 GONORRHOEA TEST-OF-CURE BY POST MAINTAINS RETURN RATE

Daniel Dennehy, Gary Whitlock, Sheel Patel, Alan McOwan, Nneka Nwokolo*. Chelsea \& Westminster Hospital, 56 Dean Street, London, UK

\subsection{6/sextrans-2015-052126.26}

Background/introduction BASHH guidelines recommend test-ofcure (TOC) in all cases of $N$. gonorrhoeae (NG) 2 weeks after treatment. Previously patients re-attended our clinic in person for TOC. To create capacity in the clinic, we introduced NG TOC postal packs for MSM following treatment.

Aim(s)/objectives To evaluate TOC return rate and patient satisfaction with the service development.

Methods MSM with proven NG were given postal TOC packs at treatment. Each pack contains appropriate NAAT sampling kits for site of diagnosed infection (rectal, throat, urine) and written instructions, patient satisfaction survey and partner notification questionnaire. Patients are instructed to return TOC samples by post in a provided Royal Mail Safebox. We processed samples using our in-house GeneXpert system; results are sent by SMS.

Results During November 2014, 136 NG TOC packs were dispensed. 76 (55.9\%) patients returned postal packs; 28 (20.6\%) attended for TOC in person, giving overall TOC rate, $76.5 \%$. NG TOC rate in October 2014 was $75.8 \%$. The median time from treatment to sending TOC results was $19 \mathrm{~d}$ (IQR:16-24d). NG TOC positivity rate was $12.5 \%$ (13/104). 65 patient satisfaction surveys were returned. Most responders found postal TOC easy to use $(81.5 \%$; 53/65). 24.6\% (16/65) responders would have preferred to attend in person for TOC.

Discussion/conclusion Postal testing is an acceptable NG TOC method which, when combined with the option to return in person, reduced unnecessary follow-up visits while maintaining TOC return rate. The high TOC positivity rate reinforces the importance of continuing to retest patients with NG after treatment.

\section{O27 HIGH RATES OF MACROLIDE RESISTANCE IN MYCOPLASMA GENITALIUM}

Rachel Pitt, Sarah Alexander*. Public Health England, London, UK

\subsection{6/sextrans-2015-052126.27}

Background/introduction Macrolide resistance has been previously reported in Mycoplasma genitalium (MG), however due to limited diagnostics, studies have been mainly restricted to specific geographical areas and small numbers of positive samples.

Aim(s)/objectives To determine the rate of macrolide resistance in MG specimens.

Methods Eighty-five MG positive specimens (72 from males, 13 from females) that had been referred for MG centralised testing (between 2010-2014), from 17 centres across England and Wales were blinded and anonymised. Specimens were then examined using a 23S rRNA PCR followed by full DNA sequence analysis. The Chi Square test was used to compare data sets.

Results $23 S$ rRNA PCR was successful in $86 \%$ (73/85) of specimens. Of the specimens examined, 84\% (61/73) harboured single nucleotide polymorphisms (SNP) associated with macrolide resistance (Table 1). Significant differences were observed between the rates of macrolide resistance in male [95\% (58/61)] and female $[25 \%(3 / 12)]$ patients $[\mathrm{P}=<0.001]$. Twelve 